\title{
Galectin-3 rs4652 A>C polymorphism is associated with the risk of gastric carcinoma and P-glycoprotein expression level
}

\author{
YI SHI ${ }^{1}$, XIANDONG LIN ${ }^{1}$, GANG CHEN $^{1,2}$, JUN YAN $^{3}$, MINGANG YING $^{3}$ and XIONGWEI ZHENG ${ }^{1,2}$ \\ Departments of ${ }^{1}$ Molecular Pathology, ${ }^{2}$ Pathology and ${ }^{3}$ Surgery, Fujian Cancer Hospital and \\ Fujian Medical University Cancer Hospital, Fuzhou, Fujian 350014, P.R. China
}

Received August 3, 2016; Accepted September 1, 2017

DOI: $10.3892 / \mathrm{ol} .2017 .7258$

\begin{abstract}
Galectin-3 serves an important function in cancer development and progression. The present study aimed to explore the association between single nucleotide polymorphisms in galectin-3, and the susceptibility to chemotherapy drug resistance of gastric carcinoma. The present study was a case-control study including 479 patients with gastric carcinoma and 458 cancer-free controls in a population from the Fujian province in Southeast China. Matrix-Assisted Laser Desorption/Ionization Time of Flight Mass Spectrometry was used to determine the genotype of rs4644 and rs4652, and immunohistochemistry was used to identify the expression level of various proteins associated with chemotherapeutic drug resistance. The results revealed that individuals exhibiting the rs4652 CA/AA genotype had a significantly increased risk of developing gastric carcinoma compared with the rs4652 CC genotype (adjusted odds ratio, 1.51; 95\% confidence interval, 1.05-2.18; adjusted $\mathrm{P}=0.03$ ). In addition, it was demonstrated that there were significant differences in the P-glycoprotein expression level depending on rs4652 genotypic distributions $\left(\chi^{2}=9.063 ; \mathrm{P}=0.028\right)$. Therefore, the present study demonstrated that rs4652 single nucleotide polymorphisms of the galectin-3 gene contribute to the susceptibility to and chemotherapeutic drug resistance of gastric carcinoma.
\end{abstract}

Correspondence to: Dr Xiongwei Zheng, Department of Molecular Pathology, Fujian Cancer Hospital and Fujian Medical University Cancer Hospital, 420 Fuma Road, Fuzhou, Fujian 350014, P.R. China

E-mail: 573925677@qq.com

Abbreviations: GC, gastric carcinoma; SNP, single nucleotide polymorphisms; MALDI-TOF MS, matrix-assisted laser desorption/ionization time-of-flight mass spectrometry; PCR, polymerase chain reaction; Pgp, P-glycoprotein; GST, glutathione S-transferase; TOPII, topoisomerase II; TS, thymidylate synthase

Key words: gastric carcinoma, galectin-3, single nucleotide polymorphisms, immunohistochemistry, chemotherapeutic drug resistance

\section{Introduction}

In 2012, there were an estimated 951,600 new gastric cancer cases and 723,100 gastric cancer-associated mortalities worldwide (1). Gastric cancer rates are typically twice as high in males as in females, and China has one of highest incidence rates (1). Cancer is a complex disease that results from interactions between multiple genetic and environmental factors $(2,3)$. Even between individuals with similar environmental risk factors, including Helicobacter pylori infection $(4,5)$, smoking (6) and obesity (7), not all individuals will develop gastric carcinoma (GC). This suggests that genetic factors may serve an important function in the development and progression of GC (8).

Galectin-3, a $31 \mathrm{kDa}$ oncogenic protein, is a member of the $\beta$-galactoside-binding gene family that regulates cell growth, proliferation, apoptosis, adhesion, metastasis and angiogenesis $(9,10)$. In GC, galectin-3 expression has been identified to be associated with metastasis; galectin-3 expression was significantly increased in metastatic lymph nodes, compared with that in the primary gastric tumor; therefore, it may be used as a biomarker for peritoneal metastasis and as a prognostic factor for gastric cancer (11-14). The silencing of galectin-3 alters the expression of a range of cancer-associated genes, including cyclin D1 and survivin, and increases the sensitivity of gastric cancer cells to chemotherapeutic agents (15). Human breast carcinoma cells that overexpress galectin-3 exhibit increased resistance to apoptosis induced by the DNA-cross-linking agent cisplatin, the anthracycline adriamycin, the anti-metabolite fluorouracil (5-FU) or the topoisomerase II inhibitor etoposide (9).

Rs4644 and rs4652 are the most common single nucleotide polymorphism (SNP) sites in galectin-3, and are associated with galectin-3 protein expression levels (16). Rs4644 and rs4652 are located in exon 3 of galectin-3. Rs $4644 \mathrm{C}>\mathrm{A}$ at position 191 results in substituting a proline for histidine (17). When rs4652 occurs at position 292, there is a threonine to proline substitution (18). Rs4644 C>A overexpression increases the rate of gastric cancer cell growth and resistance to cisplatin and 5-FU (17). The rs4644 variant of galectin-3 may exert a protective effect in prostate cancer cells (19). Furthermore, rs4644 is associated with the incidence of breast cancer, resulting in susceptibility to matrix metalloproteinase cleavage and the acquisition of resistance to drug-induced 
apoptosis (20). Rs4652 has also been identified to serve a function in glioma (21). Rs4644 and rs4652 regulate lectin galactoside-binding soluble 3 , affecting immunoglobulin binding.

Studies have demonstrated the biological and drug response alterations associated with galectin-3 SNPs in cancer (22); however, only a limited number of studies have investigated the function of galectin-3 rs4644 and rs4652 polymorphisms in the susceptibility and chemotherapeutic drug resistance of GC. Galectin-3 functional genetic variations may contribute to the development and chemotherapy response of GC. The objective of the present study was to evaluate the association between galectin-3 rs4644 and rs4652 genotypes with the risk of developing GC and its chemotherapy drug resistance in a hospital-based case-control study.

\section{Materials and methods}

Patients and controls. The study population consisted of 479 patients with primary GC undergoing total gastrectomy and 458 cancer-free individuals. The 479 patients with GC were recruited from Fujian Cancer Hospital (Fujian, China) between January 2007 and December 2009. All diagnoses were histopathologically confirmed as primary gastric cancer. The exclusion criteria were as follows: i) Patients who had previously been diagnosed with cancer; ii) any metastasis; and iii) patients who had received radiotherapy, chemotherapy or immunotherapy prior to surgery. The 458 cancer-free controls were randomly selected from a pool of healthy volunteers following a routine medical check during the same period at the Fujian cancer hospital; all volunteers had no history of personal or familial malignancy or other serious diseases. All participants provided written informed consent to participate in the study and the study was approved by the Ethics Committee of Fujian Cancer Hospital.

DNA extraction and genotyping analyses. The DNA of the patients with GC was isolated from paraffin-embedded normal stomach tissue adjacent to the tumor site (distance, $>5 \mathrm{~cm}$ ) using a QIAamp DNA FFPE Tissue kit (Qiagen $\mathrm{GmbH}$, Hilden, Germany). The DNA of cancer-free individuals was extracted from venous blood samples $(2 \mathrm{ml})$ using a Whole Blood Genome DNA Isolation kit (BioTeke Corporation, Beijing, China). The concentration of DNA was determined using a NanoDrop-1000 ultraviolet spectrophotometer, with a A260/A280 ratio between 1.8 and 2.0.

Genotyping analyses for SNPs were performed using Matrix-Assisted Laser Desorption/Ionization Time of Flight Mass Spectrometry (MALDI-TOF MS) as previously described (23). Briefly, polymerase chain reaction (PCR) was conducted to amplify the target sequence using HotStarTaq DNA Polymerase (Qiagen GmbH, Hilden, Germany). Primer sequences used for rs4644 detection were as follows: 1st-PCR primer, ACGTTGGATGTCCGGGATAAGCTCCAGGT; 2nd-PCR primer, ACGTTGGATGGCTTATCCTGGACA GGCAC; UEP_SEQ reverse primer, CTCCAGGCGCCT ACC. For rs4652 detection, 1st-PCR primer, ACGTTGGAT GAAGGAATGCCATCTCACCAG; 2nd-PCR primer, ACG TTGGATGCTACCCATCTTCTGGACAGC; UEP_SEQ reverse primer, GGACAGCCAAGTGCC.
The excess dNTPs were removed and base extension reactions were conducted using ThermoSequenase (GE Healthcare, Chicago, IL, USA). Extended products were treated with SpectroCLEAN resin (Sequenom, San Diego, CA, USA) to remove salts. Subsequently, $10 \mu 1$ of the reaction was dispensed into a SpectroCHIP microarray (Sequenom). A MALDI-TOF mass spectrometer was used to acquire the SNP profile data, which was analyzed by TYPER 4.0 software (Sequenom).

Quality control. A total of 3 control types were included: Negative control, internal standard control and repeat control. The number of negative controls and internal standard controls was $1 \%$ of the total sample number, whereas the number of repeat controls was $5 \%$ of the total sample number. For the negative control, sample DNA was replaced with water to monitor the contamination of product in the experiment. 'Chinese number one' cell line DNA was used as the internal standard control template to detect the primer design reliability of each SNP site. The 'Chinese number one' cell line is cultivated by Beijing Genomics Institute (Shenzhen, China) and the SNP site data can be accessed from the 'Chinese number one' database (24). For the repeat control, randomly selected samples were used as repeat controls to validate the stability of the experiment.

Immunohistochemical analysis. Immunohistochemical analyses were performed for 207 samples using the BenchMark XT platform (Ventana Medical Systems, Tucson, AZ, USA). Primary antibodies included P53 (Kit-0010-2, clone: DO-7), Ki67 (Kit-0005-2, clone: MIB-1), glutathione S-transferase (GST; MAB-0583, clone: LW29), P-glycoprotein (Pgp; MAB-0237, clone: C494), topoisomerase II (TOPII; MAB-0588, clone: 3F6) and thymidylate synthase (TS; MAB-0171, clone: TS106), (all ready-to-use dilution; Fuzhou Maixin Biotech, Fuzhou, Fujian, China). Each slide was subjected to heat treatment in $\mathrm{CC} 1$ retrieval solution (Ventana Medical Systems, Tucson, AZ, USA) for $30 \mathrm{~min}$ and subsequently incubated with all six antibodies at $37^{\circ} \mathrm{C}$ for $30 \mathrm{~min}$. Immune complexes were detected with the use of an UltraView 3'3-diaminobenzidine detection kit (Ventana Medical Systems, Tucson, AZ, USA) according to the manufacturer's protocol.

The sections were scored semi-quantitatively, using light microscopy, by two pathologists without any knowledge of the clinical history of the patients. The intensity of staining was evaluated according to the following scale: 0 , no staining; 1 , weak staining; 2 , moderate staining; and 3 , strong staining. The extent of staining was scored as follows: $0,<10 \% ; 1, \geq 10 \%$ and $<25 \% ; 2, \geq 25 \%$ and $<50 \% ; 3, \geq 50 \%$ and $<75 \% ; 4, \geq 75 \%$. The final results were the product of the intensity and extent of staining. The grading system was divided into four parts according to the product of the intensity and the extent of staining: 0-3, negative; 4-6, +; 7-9, 2+; and 10-12, 3+.

Statistical analysis. Univariate analysis was performed with a $\chi^{2}$ test. The computation of odds ratio (OR) and $95 \%$ confidence intervals $(95 \% \mathrm{CI})$ were conducted using SPSS software (version 17.0; SPSS, Inc., Chicago, IL, USA). The tests included an evaluation of the association between genotypic distributions and the development of GC, clinicopathological features (including tumor size, depth of invasion, differentiation and the 
Table I. Baseline data for patients with gastric carcinoma and healthy controls.

\begin{tabular}{|c|c|c|c|c|}
\hline Characteristic & Cases, n $(\%)$ & Controls, n (\%) & $\chi^{2}$ value & P-value \\
\hline \multicolumn{5}{|l|}{ Sex } \\
\hline Male & $347(72.44)$ & $335(73.14)$ & 0.33 & 0.56 \\
\hline Female & $132(27.56)$ & $117(25.55)$ & & \\
\hline \multicolumn{5}{|l|}{ Age, years } \\
\hline$\leq 60$ & $156(32.57)$ & $147(32.10)$ & 0.17 & 0.68 \\
\hline$>60$ & $323(67.43)$ & $287(62.66 \%)$ & & \\
\hline \multicolumn{5}{|c|}{ Lauren classification } \\
\hline Intestinal type & $175(36.53)$ & & & \\
\hline Diffuse type & $304(63.47)$ & & & \\
\hline \multicolumn{5}{|c|}{ Tumor-node-metastasis stage } \\
\hline $0-\mathrm{I}$ & $60(12.53)$ & & & \\
\hline II & $100(20.88)$ & & & \\
\hline III & $298(62.21)$ & & & \\
\hline IV & $21(4.38)$ & & & \\
\hline Total, n & 479 & 458 & & \\
\hline
\end{tabular}

Sex data for 6 control patients and age data for 24 patients is missing.

Table II. Association between galectin-3 polymorphism with the occurrence of gastric carcinoma.

\begin{tabular}{lccc}
\hline Genotype & Cases, n $(\%)$ & Controls, n (\%) & $\chi^{2}$ value $^{\text {P-value }}$ \\
\hline Total & 479 & 458 & 0.26 \\
rs4644 C>A & $11(2.33)$ & $16(3.59)$ & 1.28 \\
AA & $462(97.67)$ & $430(96.41)$ & $0.03^{\mathrm{a}}$ \\
AC/CC & $58(12.24)$ & $79(17.40)$ & 4.92 \\
rs4652 A $>$ C & $416(87.76)$ & $375(82.60)$ & \\
CC & & & \\
CA/AA & &
\end{tabular}

There are 6 patient and 12 control samples missing from the rs 4644 genotype group, and 5 patient and 4 control samples missing from the rs4652 genotype group. ${ }^{\mathrm{a}} \mathrm{P}<0.05$.

status of the lymph node metastasis) and the protein expression levels from the immunohistochemistry data. Haplotypes were estimated using the Expectation Maximization algorithm (25). Haplotype association with GC was determined using SHEsis, as previously described (26).

\section{Results}

Characteristics of the study population. The fundamental background data for patient cases and patient controls are summarized in Table I, and demonstrate they were adequately matched for age and sex. In the control patients, the frequency of the genotypes of the two polymorphisms were determined using the Hardy-Weinberg test, which revealed that the P-values for rs4644 and rs 4652 were 0.81 and 0.50 , respectively. These results demonstrated that the genotype distribution conforms to a Hardy-Weinberg equilibrium.
Quality control results. The present study was based on a large number of clinical samples. A total of 10 negative controls were used and analyzed to validate that the results were not false positives. The data of the 10 internal standard controls were all matched in the 'Chinese number one' database. In addition, the conformity of 47 repeat controls was $>98 \%$. Therefore, the results of the genotyping were deemed reliable.

Patients with rs4652 CA/AA are more likely to develop GC. The genotyping results of 479 patients and 458 controls are presented in Table II, and the MALDI-TOF MS spectra are presented in Fig. 1. Compared with the controls, the patients' rs4652 allelic genotype and its genotypic distribution frequency differed significantly; the rs4652 CA/AA carriers exhibited a significant increased risk of GC (adjusted-OR, 1.51; 95\% CI, 1.05-2.18; adjusted-P=0.03), which indicated that the patients with rs4652 CA/AA were more likely to develop GC. There 

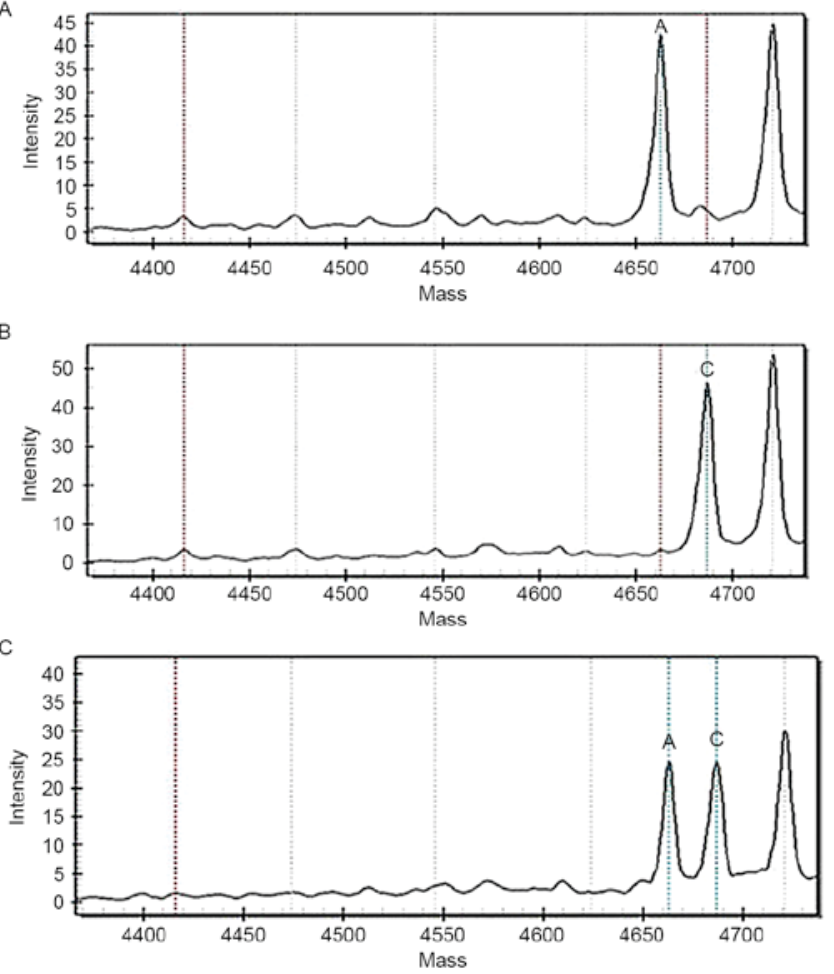

Figure 1. Genotyping diagram of rs4652 A>C. (A) rs4652 AA genotype; (B) rs4652 CC genotype; (C) rs4652 AC genotype, exhibiting peaks at sites $\mathrm{A}$ and $\mathrm{C}$.

was no significant difference identified in the genotypic distribution of rs 4644 between patients and controls, indicating that this polymorphism was not associated with the development of GC.

Galectin-3 polymorphisms are not associated with the clinicopathological features of $G C$. The present study aimed at identifying associations between the genotypic distributions of galectin-3 SNPs and the overall clinicopathological features of the disease, including tumor size, depth of invasion, differentiation and the status of lymph node metastasis. No associations were determined between rs4644 and rs4652 genotypic distribution with the overall clinicopathological features of patients with GC.

Rs4652 genotypic distributions are associated with Pgp expression. Samples from 207 patients with GC were analyzed by immunohistochemistry. Representative hematoxylin and eosin staining images of GC is presented in Fig. 2. The Pgp protein expression in the tumor tissues was predominantly homogenous and was primarily identified in the cytoplasm or at the cell membrane. With the exclusion of 4 patients who failed SNPs genotyping analyses due to low sample quality, the Pgp protein expression in the remaining 203 patients was as follows: Negative, $41.9 \%$ (85/203); +, 15.8\% (32/203); 2+, $35.0 \%$ (71/203); 3+, 7.4\% (15/203; Fig. 3). The association between the rs4652 genotypic distribution and Pgp protein expression is presented in Table III. The results indicated that there were significant associations between rs4652 genotypic distributions and Pgp expression level $\left(\chi^{2}=9.063\right.$; $\mathrm{P}=0.028)$, although the association was not linear. There was
Table III. Association between rs4652 genotypic distributions and P-glycoprotein expression.

\begin{tabular}{lrrrrr}
\hline & \multicolumn{4}{c}{ Genotypic distribution } & \\
\cline { 2 - 4 } Intensity & CC & CA & AA & CA/AA & P-value \\
\hline Negative & 61 & 25 & 0 & 25 & $0.028^{\mathrm{a}}$ \\
+ & 23 & 7 & 1 & 8 & \\
$2+$ & 49 & 22 & 0 & 22 & \\
$3+$ & 12 & 3 & 0 & 3 & \\
\hline
\end{tabular}

There is data missing from 2 patients in the negative group, 1 patient in the + group, and 1 patient in the $2+$ positive group. ${ }^{\mathrm{a}} \mathrm{P}<0.05$.

no significant association identified between the genotypic distribution of galectin-3 SNPs and other proteins including P53, Ki67, GST, Pgp, TOPII and TS.

Galectin-3 haplotype is not associated with gastric cancer risk. The haplotype of galectin-3 rs4644 and rs4652 were analyzed using SHEsis software and three haplotypes were identified in patients and controls. No association was revealed between the galectin-3 haplotype and the risk of gastric cancer.

\section{Discussion}

Previous studies have demonstrated that the expression level of galectin-3 is associated with the development and progression of gastric cancer (11-14). Galectin-3 SNPs are associated with galectin-3 expression levels (16). In addition, an association has been identified between rs 4644 and the incidence of prostate and breast cancer $(19,20)$. In the present study, no association was identified between rs4644 C>A and the susceptibility or chemotherapeutic drug resistance to GC. However, the results of the present study indicated that individuals with an rs4652 A allele (CA or AA genotype) exhibited a 1.5-fold increased risk of developing gastric cancer compared with individuals carrying the $\mathrm{CC}$ genotype.

Galectin-3 is a galactose-binding protein that belongs to the lectin family. It has three binding domains, as follows: A $\mathrm{NH}_{2}$ ligand binding domain (for target cells); a carbohydrate recognition domain and a glycine, proline, tyrosine repetitive sequence binding domain (for matrix metalloproteinase) (27). Rs4652 is located in the carbohydrate recognition domain, which is the most critical domain of galectin-3 as it activates fibroblasts via the transforming growth factor- $\beta$ pathway by ligating to fibroblast surface receptors. Activated fibroblasts (myofibroblasts) interact with cancer cells to promote angiogenesis, required for sustained cancer cell growth $(28,29)$. Therefore, we hypothesized that the rs4652 A>C alteration to the structure of the carbohydrate recognition domain strengthens its interaction with fibroblast surface receptors, resulting in the myofibroblast promotion of angiogenesis and the growth of gastric cancer cells. This hypothesis requires further study and validation.

The other principal result of the present study was that rs4652 genotypic distribution was significantly associated 

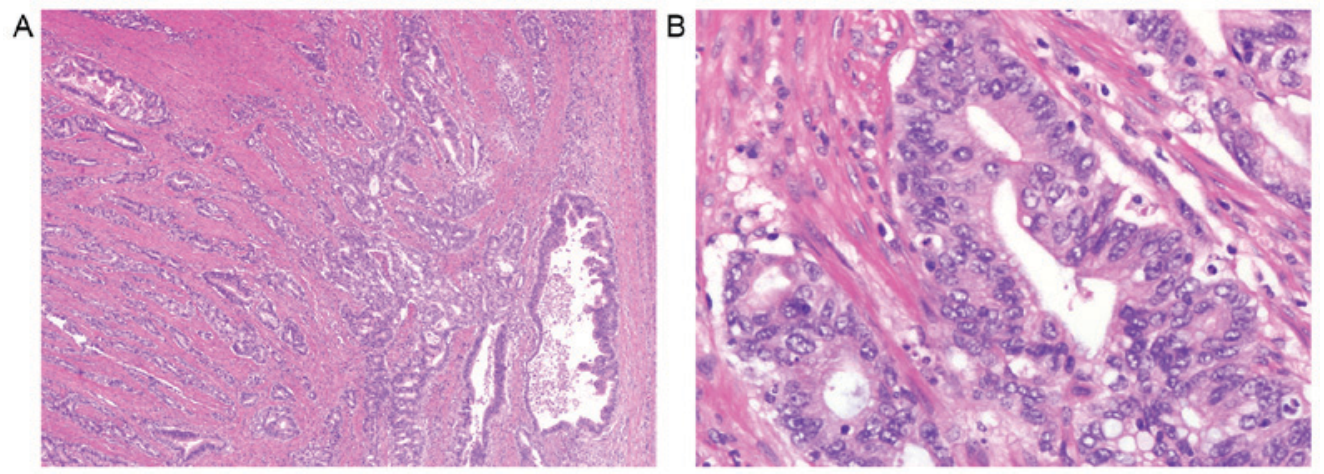

Figure 2. Hematoxylin and eosin staining of gastric carcinoma tissue. (A) Tubular adenocarcinoma infiltrating through muscle tissue. Images captured at magnification, x40. (B) Pleomorphic tumor cells reveal an epithelial-like morphology with small nuclei and symptoms of apoptosis. Images captured at magnification, $\mathrm{x} 400$.
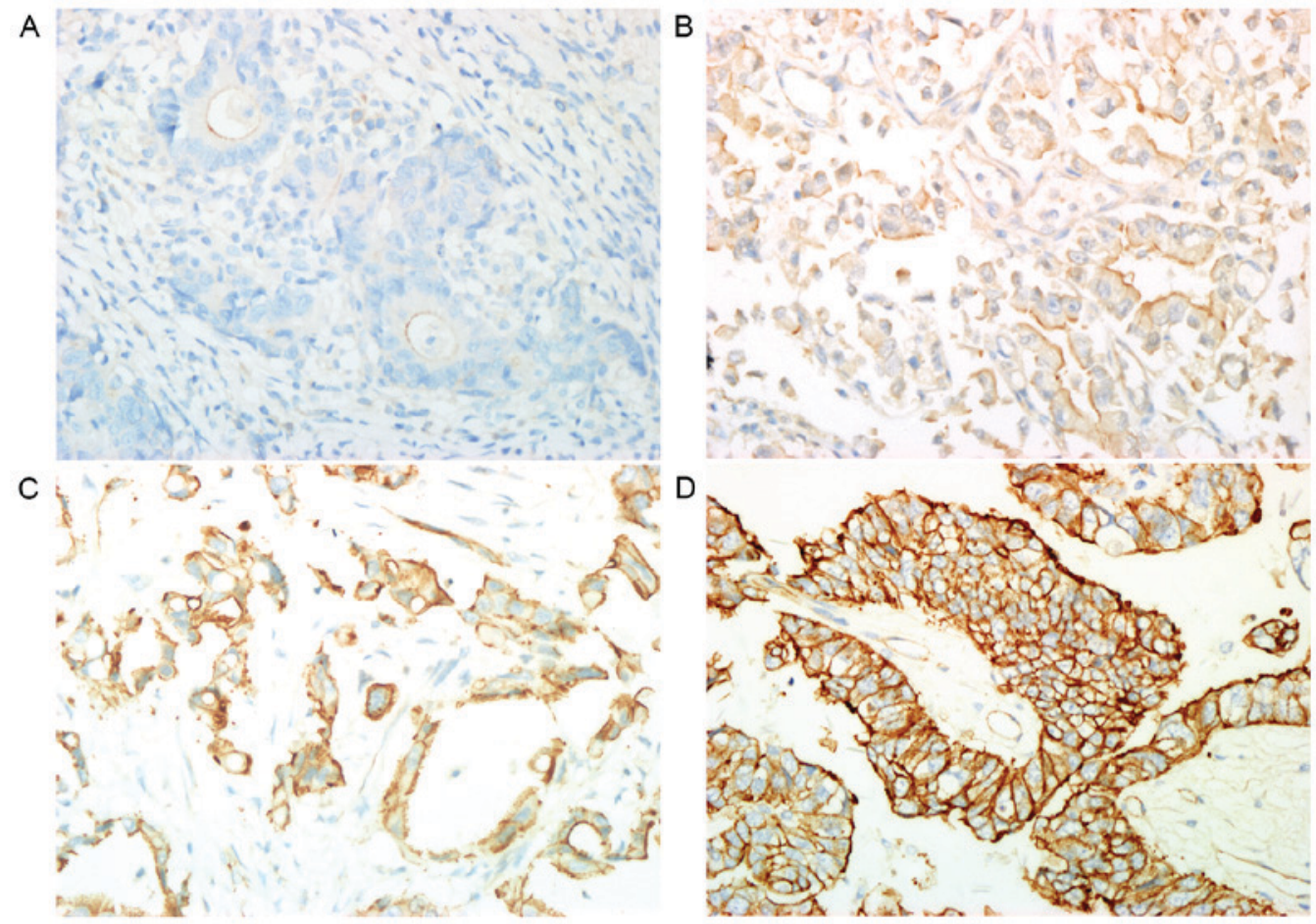

Figure 3. Representative tissue sections from 479 patients with gastric carcinoma, stained for Pgp. Representative images of tissues that were (A) Pgp negative (IHC score 0-3); (B) Pgp + (IHC score 4-6 (C) Pgp 2+ (IHC score 7-9); and (D) Pgp 3+ (IHC score 10-12). Pgp, P-glycoprotein; IHC, immunohistochemistry.

with the Pgp protein expression level, indicating that patients with GC with different genotypes may exhibit a distinct reaction to chemotherapeutic drugs. The resistance of gastric cancer cells to chemotherapeutics following surgery remains a challenge in the treatment of patients with gastric cancer. The expression of Pgp, TOPII, GST and TS are recognized as classic resistance indexes, and are associated with a poor prognosis (30). Pgp is a transmembrane protein encoded by the gene, MDR1. Pgp consists of 1,280 amino acids, with a molecular mass of $170 \mathrm{kDa}, 12$ transmembrane domains and 2 ATP binding domains. The Pgp protein binds chemotherapeutic molecules and pumps the drug out of the cell in an ATP-dependent reaction, resulting in a decreased intracellular concentration of the drug and facilitating drug resistance (31). Therefore, the increased expression of the Pgp protein can be used as a marker for multi-drug resistance. The level of Pgp protein expression has an effect on the distribution of a range of natural hydrophobic drugs, including doxorubicin, pharmorubicin, taxol and docetaxel (32). Therefore, drugs should be carefully selected for patients according to Pgp expression level. Although Pgp expression level was associated with rs4652 genotypic distribution in the present study, no linear relationship was identified; therefore, it cannot be concluded that a particular genotype is associated with increased Pgp protein expression or chemotherapeutic resistance.

The present study included certain limitations. First, data on environmental factors, including Helicobacter pylori or Epstein-Barr virus infection, were not available; therefore, it was not possible to explore gene-environment interactions. In addition, there was a lack of follow-up data; analyses to determine the function of galectin-3 polymorphisms in GC prognosis could not be performed. 
The results of the present study suggested that rs4652 SNPs of the galectin-3 gene contribute to the susceptibility to and chemotherapeutic drug resistance of GC. Thus, galectin-3 rs4652 SNPs may be a biomarker for predicting the development and prognosis of patients with GC.

\section{Acknowledgements}

The present study was supported by the National Natural Science Foundation (grant no. 81470111), the Natural Science Foundation of Fujian Province (grant no. 2014J01300) and the National Clinical Key Specialty Construction Program of China.

\section{References}

1. Torre LA, Bray F, Siegel RL, Ferlay J, Lortet-Tieulent J and Jemal A: Global cancer statistics, 2012. CA Cancer J Clin 65: 87-108, 2015

2. Liu L, Zhong R, Wei S, Yin JY, Xiang H, Zou L, Chen W, Chen JG, Zheng XW, Huang LJ, et al: Interactions between genetic variants in the adiponectin, adiponectin receptor 1 and environmental factors on the risk of colorectal cancer. PLoS One 6: e27301, 2011.

3. Reeves GK, Pirie K, Green J, Bull D and Beral V; Million Women Study Collaborators: Comparison of the effects of genetic and environmental risk factors on in situ and invasive ductal breast cancer. Int J Cancer 131: 930-937, 2012.

4. Parkin DM: The global health burden of infection-associated cancers in the year 2002. Int J Cancer 118: 3030-3044, 2006

5. Plummer M, Franceschi S, Vignat J, Forman D and de Martel C: Global burden of gastric cancer attributable to Helicobacter pylori. Int J Cancer 136: 487-490, 2015.

6. Bertuccio P, Chatenoud L, Levi F, Praud D, Ferlay J, Negri E, Malvezzi M and La Vecchia C: Recent patterns in gastric cancer: A global overview. Int J Cancer 125: 666-673, 2009.

7. Jemal A, Center MM, DeSantis C and Ward EM: Global patterns of cancer incidence and mortality rates and trends. Cancer Epidemiol Biomarkers Prev 19: 1893-1907, 2010.

8. Lin XD, Chen SQ, Qi YL, Zhu JW, Tang Y and Lin JY: Polymorphism of THBS1 rs1478604 A $>\mathrm{G}$ in 5-untranslated region is associated with lymph node metastasis of gastric cancer in a Southeast Chinese population. DNA Cell Biol 31: 511-519, 2012.

9. Fukumori T, Kanayama HO and Raz A: The role of galectin-3 in cancer drug resistance. Drug Resist Updat 10: 101-108, 2007.

10. Nakahara S, Oka N and Raz A: On the role of galectin-3 in cancer apoptosis. Apoptosis 10: 267-275, 2005

11. Jeong GA, Kim HC, Kim HK and Cho GS: Perigastric lymph node metastasis from papillary thyroid carcinoma in a patient with early gastric cancer: The first case report. J Gastric Cancer 14: 215-219, 2014.

12. Lee KB, Park DJ, Choe G, Kim HH, Kim WH and Lee HS: Protein expression status in mucosal and submucosal portions of early gastric cancers and their predictive value for lymph node metastasis. APMIS 121: 926-937, 2013.

13. Yang ZM, Wu XT, He T, Da MX, Luo T and Qian K: Study on association between the expression of galectin- 3 and the peritoneal metastasis in gastric cancer. Zhonghua Wei Chang Wai Ke Za Zhi 8: 151-154, 2005 (In Chinese).

14. Miyazaki J, Hokari R, Kato S, Tsuzuki Y, Kawaguchi A, Nagao S, Itoh K and Miura S: Increased expression of galectin-3 in primary gastric cancer and the metastatic lymph nodes. Oncol Rep 9: 1307-1312, 2002.
15. Cheong TC, Shin JY and Chun KH: Silencing of galectin-3 changes the gene expression and augments the sensitivity of gastric cancer cells to chemotherapeutic agents. Cancer Sci 101: 94-102, 2010.

16. de Boer RA, Verweij N, van Veldhuisen DJ, Westra HJ, Bakker SJ, Gansevoort RT, Muller Kobold AC, van Gilst WH, Franke L, Mateo Leach I and van der Harst P: A genome-wide association study of circulating galectin-3. PLoS One 7: e47385, 2012.

17. Kim SJ, Shin JY, Cheong TC, Choi IJ, Lee YS, Park SH and Chun KH: Galectin-3 germline variant at position 191 enhances nuclear accumulation and activation of $\beta$-catenin in gastric cancer. Clin Exp Metastasis 28: 743-750, 2011.

18. Hu CY, Chang SK, Wu CS, Tsai WI and Hsu PN: Galectin-3 gene (LGALS3) +292C allele is a genetic predisposition factor for rheumatoid arthritis in Taiwan. Clin Rheumatol 30: 1227-1233, 2011.

19. Mazurek N, Byrd JC, Sun Y, Ueno S and Bresalier RS: A galectin-3 sequence polymorphism confers TRAIL sensitivity to human breast cancer cells. Cancer 117: 4375-4380, 2011.

20. Balan V, Nangia-Makker P, Schwartz AG, Jung YS, Tait L, Hogan V, Raz T, Wang Y, Yang ZQ, Wu GS, et al: Racial disparity in breast cancer and functional germ line mutation in galectin-3 (rs4644): A pilot study. Cancer Res 68: 10045-10050, 2008.

21. Lee YH and Song GG: Genome-wide pathway analysis in glioma. Neoplasma 62: 230-238, 2015.

22. Song L, Tang JW, Owusu L, Sun MZ, Wu J and Zhang J: Galectin-3 in cancer. Clin Chim Acta 431: 185-191, 2014.

23. Justenhoven C, Hamann U, Pesch B, Harth V, Rabstein S, Baisch C, Vollmert C, Illig T, Ko YD, Brüning T and Brauch H: ERCC2 genotypes and a corresponding haplotype are linked with breast cancer risk in a German population. Cancer Epidemiol Biomarkers Prev 13: 2059-2064, 2004.

24. Wang J, Wang W, Li R, Li Y, Tian G, Goodman L, Fan W, Zhang J, Li J, Zhang J, et al: The diploid genome sequence of an Asian individual. Nature 456: 60-65, 2008.

25. Li Z, Zhang Z, He Z, Tang W, Li T, Zeng Z, He L and Shi Y: A partition-ligation-combination-subdivision EM algorithm for haplotype inference with multiallelic markers: Update of the SHEsis (http: //analysis.bio-x.cn). Cell Res 19: 519-523, 2009.

26. Shi YY and He L: SHEsis, a powerful software platform for analyses of linkage disequilibrium, haplotype construction, and genetic association at polymorphism loci. Cell Res 15: 97-98, 2005.

27. Zhou JP, Yang ZL, Liu DC and Zhou JP: Expression of galectin 3 and Sambucus nigra agglutinin and their clinicopathological significance in benign and malignant lesions of stomach. Zhonghua Wei Chang Wai Ke Za Zhi 12: 297-300, 2009 (In Chinese).

28. Miyazono K, Ehata S and Koinuma D: Tumor-promoting functions of transforming growth factor- $\beta$ in progression of cancer. Ups J Med Sci 117: 143-152, 2012.

29. Webber JP, Spary LK, Sanders AJ, Chowdhury R, Jiang WG, Steadman R, Wymant J, Jones AT, Kynaston H, Mason MD, et al: Differentiation of tumour-promoting stromal myofibroblasts by cancer exosomes. Oncogene 34: 290-302, 2015.

30. Shi H, Lu D, Shu Y, Shi W, Lu S and Wang K: Expression of multidrug resistance-related proteins p-glycoprotein, glutathione-s-transferases, topoisomerase-II and lung resistance protein in primary gastric cardiac adenocarcinoma. Hepatogastroenterology 55: 1530-1536, 2008.

31. Sui H, Zhou S, Wang Y, Liu X, Zhou L, Yin P, Fan Z and Li Q: COX-2 contributes to P-glycoprotein-mediated multidrug resistance via phosphorylation of c-Jun at Ser63/73 in colorectal cancer. Carcinogenesis 32: 667-675, 2011.

32. Roy S, Kenny E, Kennedy S, Larkin A, Ballot J, Perez De Villarreal M, Crown J and O'Driscoll L: MDR1/P-glycoprotein and MRP-1 mRNA and protein expression in non-small cell lung cancer. Anticancer Res 27: 1325-1330, 2007. 